\title{
Optimización de parámetros en procesos de fundición a presión y compresión mediante el algoritmo de enjambre de partículas
}

\author{
Optimization of parameters in die casting and compression processes \\ using the particle swarm algorithm
}

Yordy González-Rondón ${ }^{1}$, José Eduardo Rengel ${ }^{2}$, Johnny J. Martínez ${ }^{3}$

Recibido: Febrero 2021

Aceptado: Agosto 2021

Resumen.- En este artículo, se desarrolló un algoritmo, basado en la técnica de enjambre de partículas (Siglas en inglés - PSO) para optimizar los procesos de fundición a presión y por compresión, utilizando modelos matemáticos para describir el comportamiento de ambos procesos. En la fundición por compresión el modelo matemático describe un problema con múltiples objetivos y restricciones, y en la fundición a presión el modelo describe un problema de un solo objetivo con restricciones. El desarrollo del algoritmo PSO se realizó con el software FORTRAN 90, y los resultados se compararon con los obtenidos usando un algoritmo de optimización basado en el proceso de enseñanza-aprendizaje (Siglas en inglés - TLBO), demostrando buenas capacidades en la optimización de parámetros en fundición a presión y por compresión. Se observó que con el algoritmo PSO se consigue una solución óptima en todos los procesos y los tiempos computacionales fueron mínimos.

Palabras clave: Optimización de parámetros, fundición por compresión, fundición a presión, algoritmo PSO.

Summary.- In this article, an algorithm, based on the particle swarm technique (PSO), is developed to optimize die casting and compression casting processes, using mathematical models to describe the behavior of both processes. In compression casting the mathematical model describes a problem with multiple objectives and constraints, and in die casting the model describes a single objective problem with constraints. The development of the PSO algorithm was carried out with the FORTRAN 90 software, and the results were compared with those reported by a teaching-learning based optimization algorithm, (TLBO), demonstrating good capabilities in the optimization of parameters in die casting and by compression. It was observed that the PSO algorithm achieves an optimal solution in all processes and the computational time were minimal.

Keywords: Parameter optimization, Squeeze casting, Die casting, PSO algorithm

\footnotetext{
${ }^{1}$ Ingeniería Mecánica, Universidad de Oriente (Venezuela), yordygonzalez@hotmail.com, ORCID iD: https://orcid.org/0000-0002-4769-7568

22Ingeniería Mecánica, Universidad de Oriente (Venezuela), Rengel66@gmail.com, ORCID iD: https://orcid.org/0000-0002-4510-8846

${ }^{3}$ Ingeniería Mecánica, Universidad de Oriente (Venezuela), johnnymartinr@gmail.com, ORCID iD: https://orcid.org/0000-0001-8901-9303
} 
1. Introducción. - Desde la antigüedad se ha utilizado la fundición para la fabricación de piezas de diversas formas, el proceso consiste en llevar a muy altas temperaturas la materia prima (hierro, acero, aluminio, zinc, magnesio, entre otros) y vaciar en un molde donde se deja enfriar [1]. Actualmente se conocen distintos métodos de fundición, una forma de clasificarlos es según el tipo de molde con la forma de llenar el fundido, y entre ellos se encuentran las fundiciones a presión y compresión [2]. Estos procesos de fundición se diferencian por la metodología que utilizan para la fabricación de las piezas, y por los diferentes parámetros de entrada que afectan la salida del producto final. En el caso de la fundición por compresión, el proceso consiste en introducir una cierta cantidad de fundido pre-especificada en una cavidad de matriz precalentada, donde solidifica bajo presión hidrostática entre las placas de una prensa hidráulica [3, 4]. El aumento de la presión en el proceso de fabricación permite alcanzar una buena calidad en la pieza fundida en términos de una superficie más lisa y mejores propiedades mecánicas [5]. Aunado a lo mencionado anteriormente, con el método por compresión se consigue una estructura de grano equiaxial [6,7], y lo que es más importante, elimina la porosidad de contracción macro/micro [8], ofreciendo importantes oportunidades para aplicaciones en una diversidad de áreas, particularmente en la industria mecánica automotriz y aeroespacial [9]. Investigaciones han surgido para entender lo que sucede en el proceso de compresión, Han et al. [10], observaron que mientras la solidificación de una aleación de $\mathrm{Al}-\mathrm{Cu}$ ocurre a alta presión, se logra un significativo refinamiento de la microestructura. Maeng et al. [11], también observaron mayor refinamiento de la microestructura debido a la presión, y un aumento tanto en la dureza como en la resistencia a la tracción. Otros estudios [12-14] han descrito que, al aumentar la presión junto con el refinamiento de la microestructura, pueden ocurrir otras modificaciones estructurales como la contracción y la macrosegregación. Por su parte, la fundición a presión es un proceso versátil que consiste en forzar el fundido a alta presión en moldes de acero reutilizables que tienen la forma deseada de la pieza $[15,16]$. La técnica a presión adquiere mayor importancia cuando se requiere una producción en masa $[15,17]$, y entre los materiales fundidos que más se utilizan, el aluminio ocupa los primeros lugares, y su aplicación abarca la fabricación de componentes en el área de ingeniería, aeronáutica y automotriz [16]. En la fundición a presión, los parámetros del proceso comúnmente a controlar son la temperatura del troquel, el tiempo de llenado del troquel, las velocidades del émbolo y de la compuerta, y la presión de intensificación [18]. Al igual que en la fundición por compresión, los parámetros del proceso que intervienen en la fundición a presión afectan la resistencia del producto y la calidad, llegando a formar defectos como la porosidad, razón por la que es indispensable calibrar las variables que intervienen en los procesos, porque una mínima variación en las magnitudes de los parámetros de entrada se verá reflejado en las características que determinan la calidad final de la pieza fabricada [19]. En este sentido, las plantas de fundición utilizan comúnmente, el método de ensayo y error para determinar estos parámetros, sin embargo, este tipo de procedimiento requiere invertir en largos periodos de trabajo experimental y traen como consecuencia pérdidas de tiempo y dinero [2]. Han surgido otras técnicas para el ajuste de parámetros en el área de la ingeniería, procesos, medicina, entre otros, denominadas Metaheurísticas de Optimización, como una alternativa para encontrar la mejor solución, o al menos una solución (incluso en muchos casos la solución óptima) en el ajuste de los parámetros de un problema [20]. Dentro de estas técnicas se encuentran los algoritmos basados en cúmulos de partículas, conocido como la técnica de Optimización por Enjambre de Partículas (PSO, por sus siglas en inglés) [21, 22], técnica bastante novedosa perteneciente a la familia de los algoritmos Bioinspirados [23] (donde también están incluidos los Algoritmos Evolutivos [24], Colonias de Hormigas [25] y optimización basada en Enseñanza-Aprendizaje (TLBO, por su siglas en inglés) [26-28]), que resuelven de manera muy efectiva y eficiente un gran conjunto de problemas de optimización de alta complejidad. La TLBO se basa en el efecto de la influencia de un profesor en el aprendizaje de los alumnos en una clase, donde un grupo de alumnos se considera población y los diferentes temas ofrecidos a los alumnos se consideran los diferentes parámetros de diseño, y 
el resultado de un alumno es análogo al valor de "aptitud" del problema de optimización [2]. La PSO es inspirado en el comportamiento social del vuelo de las bandadas de aves o el movimiento de los bancos de peces que simula la toma de decisión individual y grupal para conseguir un objetivo común que es la comida [21].

De manera de conocer el estado del arte en la aplicación de la PSO en procesos de fundición, en este estudio se realizó una búsqueda bibliográfica que evidenciara los esfuerzos realizados en la aplicación de algoritmos de optimización PSO para al ajuste de parámetros en modelos de simulación respecto a las fundiciones a presión y compresión, sin embargo, los resultados fueron escasos. Entre estos, Patel et al. [29], refiriéndose a un proceso de fundición por compresión, utilizaron tres métodos de búsqueda y optimización de parámetros: algoritmo genético (GA, por sus siglas en ingles), optimización del enjambre de partículas (PSO) y optimización del enjambre de partículas multiobjetivo (MOPSO-CD). Los resultados obtenidos indicaron que el PSO superó al GA en tiempo de cálculo. Shu Fu-hua [30], combinó PSO y red neuronal artificial en la optimización de los parámetros de un proceso de fundición por compresión, y determinó que el algoritmo desarrollado fue capaz de proporcionar buenos resultados en cuanto a los parámetros del proceso, los cuales permitieron alcanzar propiedades mecánicas con estrecha concordancia a las propiedades mecánicas previstas. Panicker y Kuriakose [31], utilizaron un algoritmo metaheurístico mejorado llamado DHOA basado en un coeficiente modificado (MC-DHOA) y un algoritmo PSO, para la optimización de parámetros de un proceso de fundición a compresión, donde llegaron a la conclusión que el rendimiento general del MC-DHOA fue un 16,5\% mejor que el PSO. Wu et al. [32], utilizaron datos experimentales de una matriz ortogonal L25 del método Taguchi como muestras de entrenamiento para examinar la porosidad de una pieza fundida a presión en relación con los parámetros de las variables de fundición. Ellos también usaron un algoritmo PSO para optimizar el modelo y minimizar la porosidad. Los resultados demostraron que la PSO superó al método Taguchi, proporcionando una mejora significativa sobre los resultados de la optimización. Patel, et al. [33], desarrollaron un código de optimización basada en la enseñanza-aprendizaje (TLBO) para optimizar los parámetros de entrada-salida de un proceso de fundición por compresión. Compararon los resultados del TLBO con varios algoritmos evolutivos (GA, PSO, entre otros.) y determinaron que el TLBO superó a los algoritmos evolutivos en tiempo de cálculo.

La revisión bibliográfica dio a conocer que aún falta mucho por avanzar en la aplicación del algoritmo PSO en los procesos de fundición a presión y compresión, para comprobar la efectividad del algoritmo en estos tipos de procesos. Además, los estudios que han antecedido a esta investigación no son suficientes como para dar una valoración definitiva de la eficacia del PSO. En este sentido, en la presente investigación se desarrolló un algoritmo PSO aplicable a los procesos de fundición a presión y compresión descrito por Venkata et al. [2], quienes demostraron la aplicabilidad de un algoritmo de optimización basado en enseñanza-aprendizaje (TLBO). A su vez, el trabajo de Venkata et al. [2], utilizó como referencia los trabajos de Senthil y Amirthagadeswaran [34] y Tsoukalas [35], quienes hicieron uso de métodos de optimización experimentales y algoritmo genético. Los resultados de esta investigación reportaron que la PSO, respecto al TLBO, tiene buenas capacidades en la optimización de parámetros en fundición a presión y por compresión, consiguiendo una solución óptima en todos los procesos y con esfuerzos computacionales mínimo. En próximas investigaciones se pretende aplicar la PSO en otros métodos de fundición como lo es la colada continua de acero.

2. Diseño de la técnica de optimización por enjambre de partículas (PSO). En este artículo se diseñó una técnica de optimización para el ajuste de parámetros en los procesos de fundición a presión y compresión basándose en algoritmos metaheurísticos, para proveer una solución aceptable en relación al tiempo para solucionar un problema difícil y complejo en la rama de la ciencia y la ingeniería [36]. La metaheurística que se ha convenido describir se basa en Enjambre 
de Partículas o Particle Swarm Optimization (PSO). La optimización por enjambre de partículas fue introducida por Kennedy y Eberhart en 1995 [21, 22], inspirados en el comportamiento social del vuelo de las bandadas de aves o el movimiento de los bancos de peces que simula en base a la siguiente declaración [37]:

"Ningún pájaro sabe dónde está la comida; pero cada uno sabe a qué distancia se encuentra de la comida y también la distancia de sus compañeros a la comida. Entonces los pájaros se mueven hacia el más cercano a la comida durante un tiempo. Luego, cada pájaro vuelve a examinar su distancia a la comida y también la de sus compañeros, y así se mueven hacia el pájaro más cercano a la comida. El examen y el movimiento se repiten en varias iteraciones hasta encontrar la comida"

En este sentido, la PSO que se diseñó en este estudio, se fundamentó en los factores que influyen en la toma de decisión de una partícula que forma parte de un conjunto de partículas similares. La toma de decisión por parte de cada partícula se realiza conforme a una componente social y una componente individual, mediante las cuales se determina el movimiento (dirección) de esta partícula para alcanzar una nueva posición en el espacio de soluciones [21, 22]. La componente individual puede modificar su opinión dependiendo de:

- Su conocimiento sobre el entorno (su valor de fitness).

- Su conocimiento histórico o experiencias anteriores (su memoria o conocimiento cognitivo).

- El conocimiento histórico o experiencias anteriores de las partículas situadas en su entorno (su conocimiento social).

En base a éstos, cada partícula adapta su conjunto de creencias según las creencias de aquellas con más éxitos de su entorno, originando así una cultura en dónde el enjambre de partículas tiene un conjunto de creencias estrechamente relacionadas. Este enjambre de partículas puede verse como un sistema multiagente, dónde las partículas son agentes simples que se mueven por el espacio de búsqueda, guardando y comunicando la mejor solución que hayan encontrado.

Cada partícula tiene un fitness, una posición y un vector velocidad que dirige su movimiento. El movimiento de las partículas por el espacio está guiado por las partículas óptimas en el momento actual. Cada partícula, $p_{i}$, está compuesta por tres vectores y dos valores fitness [38], tal como se especifica a continuación:

- El vector $x_{i}=\left(x_{i 1}, x_{i 2}, \ldots, x_{i n}\right)$ almacena la posición actual de la partícula en el espacio de búsqueda.

- El vector $p B e s t_{i}=\left(p_{i 1}, p_{i 2}, \ldots, p_{i n}\right)$ almacena la posición de la mejor solución encontrada por la partícula hasta el momento.

- El vector velocidad $v_{i}=\left(v_{i 1}, v_{i 2}, \ldots, v_{i n}\right)$ almacena el gradiente (dirección) según se mueva la partícula.

- El valor de fitness fitness_ $x_{i}$ almacena el valor de aptitud de la solución actual (vector $x_{i}$ ).

- El valor de fitness fitness_pBest $t_{i}$ almacena el valor de aptitud de la mejor solución local encontrada hasta el momento (vector $p$ Best $_{i}$ ).

En el tiempo, la partícula actualiza su posición de la siguiente forma:

$$
x_{i}(t+1)=x_{i}(t)+v_{i}(t+1)
$$

donde $x_{i}(t+1)$ es la posición futura o bien la posición actualizada, $x_{i}(t)$ representa la posición de la partícula en el instante de tiempo $t$, y $v_{i}(t+1)$ es la velocidad actualizada de la partícula $i$. La figura I muestra gráficamente el movimiento de la partícula. 
Debido a que el vector velocidad se modifica teniendo en cuenta su experiencia y la de su entorno, la expresión que lo describe será la siguiente:

$$
v_{i}(t+1)=w v_{i}(t)+c_{1} \operatorname{rand}_{1}\left(p \text { Best }_{i}-x_{i}(t)\right)+c_{2} \operatorname{rand}_{2}\left(g B e s t_{i}-x_{i}(t)\right)
$$

donde $w$ representa el factor de inercia que representa el grado de influencia de la velocidad actual sobre la velocidad futura, los factores $c_{1}$ y $c_{2}$ son constantes de aceleración cognitiva y social, que determinan en qué medida influyen sobre el movimiento de la partícula su propia memoria y la cooperación entre individuos, respectivamente, $\operatorname{rand}_{1}$ y $\operatorname{rand}_{2}$ son dos números aleatorios uniformemente distribuidos entre 0 y 1 cuyo objetivo es emular el comportamiento estocástico y un tanto impredecible que exhibe la población del enjambre, y $g B e s t_{i}$ representa la posición de la mejor solución encontrada por todo el cúmulo. El valor del factor inercia en función del tiempo está dada por la ecuación (3) [39]:

$$
w(t)=w_{\max }-\frac{w_{\max }-w_{\min }}{t_{\max }} t
$$

donde $w_{\max } \mathrm{y} w_{\min }$ son los valores máximo y mínimo de $w, \mathrm{y} t_{\max }$ es el número máximo de iteraciones.

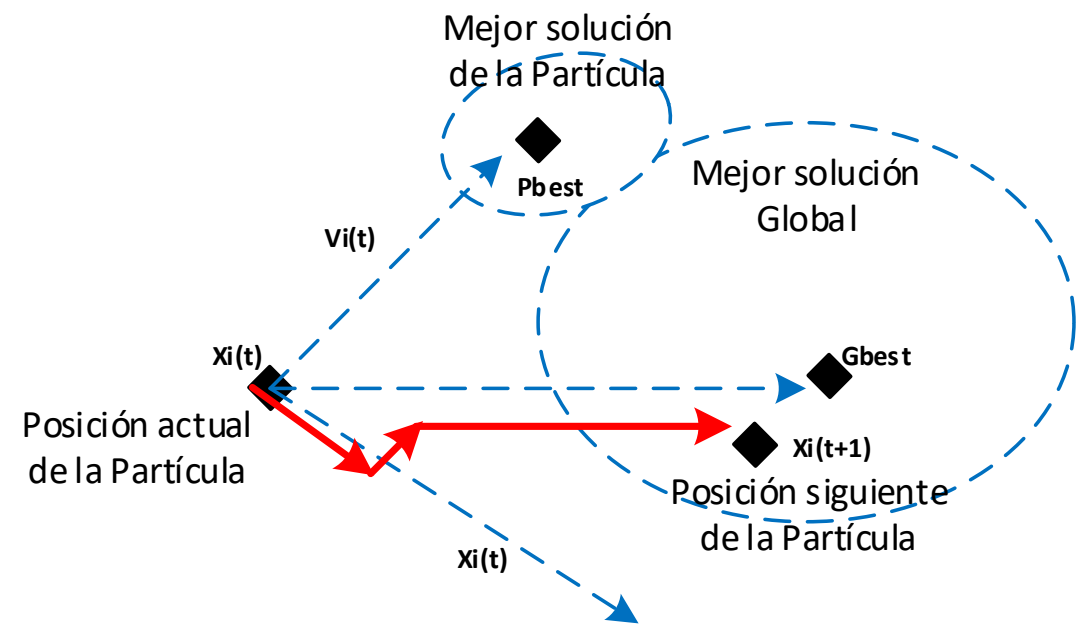

Figura I. Movimiento de una partícula en el espacio de soluciones [20]

Conceptualmente, los tres términos que componen la velocidad representan [40]:

- Inercia o impulso $\left(w v_{i}(t)\right)$ : previene de que la partícula cambie drásticamente de dirección, ya que mantiene un registro de la orientación anterior.

- Componente cognitivo $\left(c_{1} \operatorname{rand}_{1}\left(\right.\right.$ pBest $\left.\left._{i}-x_{i}(t)\right)\right)$ : tiene en cuenta la tendencia de la partícula para volver a la mejor posición previamente encontrada por ella misma.

- Componente social $\left(c_{2} \operatorname{rand}_{2}\left(g\right.\right.$ Best $\left.\left._{i}-x_{i}(t)\right)\right)$ : determina la capacidad de la partícula de moverse hacia la mejor posición encontrada por todo el enjambre.

La información presentada en este apartado muestra la metodología de funcionamiento del algoritmo de optimización por enjambre de partículas desarrollado en este estudio. El diagrama de 
flujo que se describe en la Figura II proporciona un mayor entendimiento de cómo funciona el algoritmo de la PSO diseñado, el cual se codificó en el software Fortran 90. Se inicia con una población, con posiciones y velocidades aleatorias (dentro de los límites especificados). Posteriormente, se calcula la función de adaptación de cada partícula. Luego si no se cumple los criterios establecidos de parada, se procede a calcular la mejor posición que ha tenido cada partícula $\left(p B e s t_{i}\right)$ y la mejor posición entre el grupo de partículas $\left(g B e s t_{i}\right)$, para actualizar los individuos utilizando las ecuaciones (1) y (2). El procedimiento se repite hasta cumplir el criterio de parada referente al máximo de iteraciones. Al finalizar, en pantalla se muestran los resultados correspondientes a los parámetros optimizados y los objetivos alcanzados.

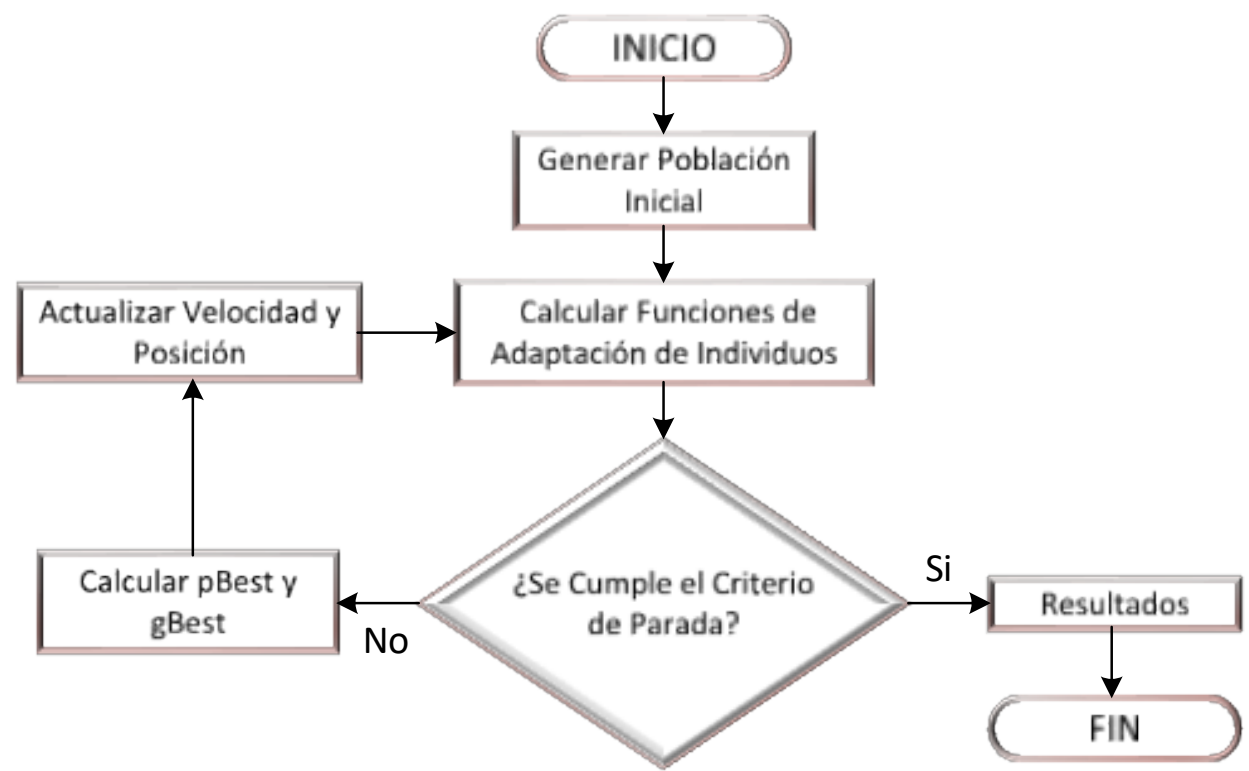

Figura II. Diagrama de flujo de la PSO [39]

Con la finalidad de estudiar la aplicabilidad y efectividad del algoritmo de enjambre de partículas propuesto en este trabajo, específicamente en los procesos de fundición a presión y compresión, se utilizaron los modelos matemáticos propuesto por Senthil y Amirthagadeswaran [34] que describen una fundición a compresión y los modelos de Tsoukalas [35] que describen una fundición a presión, para simular el ajuste adecuado de los parámetros que se manejan en estos procesos, además de determinar si estos parámetros conllevan a conseguir una mayor dureza y resistencia a la tracción en la fundición a compresión, y una mínima porosidad en la función a presión. Los resultados aquí reportados, fueron comparados con los obtenidos por Venkata et al. [2], en la sección de resultados y discusión.

\section{Resultados y Discusión.-}

3.1 Optimización de parámetros en el proceso de fundición por compresión.- Senthil y Amirthagadeswaran [34], en su investigación prepararon fundiciones de aleación de aluminio para estudiar experimentalmente la influencia de la presión, así como otros parámetros del proceso, sobre las propiedades mecánicas. Aplicaron la técnica de Taguchi para determinar el número requerido de experimentos y los parámetros del proceso fueron la presión de compresión $(A)$, la temperatura de fusión $(B)$, la temperatura de precalentamiento del troquel $(C)$, y el tiempo de 
compresión $(E)$. Con el Software MINITAB 14 desarrollaron modelos matemáticos para la dureza $(\mathrm{H}$, en $\mathrm{BHN})$ y la resistencia a la tracción (TS, en MPa), y los resultados fueron los siguientes:

$$
\begin{gathered}
H=-3,82542+0,8787 * A+0,46587 * C+0,30411 * E-0,00393 * A^{2} \\
\quad-0,00116 \\
* C^{2}+0,00097 * E^{2}+0,00051 * A * C-0,00333 * A * E-0,00018 * C * E \\
T S=-11,2606+2,5778 * A+1,3316 * C+0,7552 * E-0,0116 * A^{2} \\
\quad-0,0034 * C^{2} \\
+0,0031 * E^{2}+0,0015 * A * C-0,0097 * A * E-0,001 * C * E
\end{gathered}
$$

Los rangos de los parámetros del proceso utilizados fueron:

$$
\begin{gathered}
A=(50-125) M P a \\
B=(675-750)^{\circ} \mathrm{C} \\
C=(150-300)^{\circ} \mathrm{C} \\
E=(15-60) s
\end{gathered}
$$

Los valores óptimos que reportaron Senthil y Amirthagadeswaran [34] del proceso de fundición por compresión fueron los siguientes: $A=100 \mathrm{MPa}, B=725^{\circ} \mathrm{C}, C=200{ }^{\circ} \mathrm{C}$ y $E=45 \mathrm{~s}$. Con estos parámetros obtuvieron una máxima dureza de $100,76 \mathrm{BHN}$ y una máxima resistencia a la tracción de 278,45 MPa.

Posteriormente, Venkata et al. [2], desarrollaron un algoritmo de optimización basado en enseñanza-aprendizaje (TLBO), utilizando los modelos descritos por Senthil y Amirthagadeswaran [34]. El algoritmo TLBO fue ejecutado con un tamaño de población de 10, un número de iteraciones de 20 , y para $A=119 \mathrm{MPa}, B=686^{\circ} \mathrm{C}, C=225^{\circ} \mathrm{C}$ y $E=15 \mathrm{~s}$, la dureza obtenida fue de $103,068 \mathrm{BHN}$, y cuando $A=119 \mathrm{MPa}, B=675^{\circ} \mathrm{C}, C=220^{\circ} \mathrm{C}$ y $E=15 \mathrm{~s}$, la resistencia

\begin{tabular}{|c|c|c|c|c|c|}
\hline \multirow[b]{2}{*}{ Parámetros } & \multirow[b]{2}{*}{ Exp. } & \multirow[b]{2}{*}{ TLBO } & \multicolumn{3}{|c|}{ PSO } \\
\hline & & & Resultados & Media & $\begin{array}{c}\text { D. } \\
\text { Estándar }\end{array}$ \\
\hline Presión de compresión (MPa) & 100 & 119 & 120 & & \\
\hline Temperatura de fusión $\left({ }^{\circ} \mathrm{C}\right)$ & 725 & 686 & 713 & & \\
\hline Temperatura de compresión $\left({ }^{\circ} \mathrm{C}\right)$ & 200 & 225 & 226 & & \\
\hline Tiempo de compresión (s) & 45 & 15 & 15 & & \\
\hline Máxima dureza(BHN) & 100,76 & 103,068 & 103,072 & 103,071 & 0,001 \\
\hline
\end{tabular}
máxima a la tracción obtenida fue de $290,30 \mathrm{MPa}$, lo que representó una mejora de los resultados de Venkata et al. [2] respecto al estudio de Senthil y Amirthagadeswaran [34].

Ahora, en el presente estudio, se ejecutó el código basado en la técnica de optimización de enjambre de partículas, utilizando las restricciones y modelos matemáticos descritos por Senthil y Amirthagadeswaran [34]. Se realizaron 50 ejecuciones con el código PSO, cada una con 20 iteraciones y una población de 50 , para $A=120 \mathrm{MPa}, B=713^{\circ} \mathrm{C}, \mathrm{C}=226^{\circ} \mathrm{C}$ y $E=15 \mathrm{~s}$, se obtuvo una dureza de $103,072 \mathrm{BHN}$, y cuando $A=119 \mathrm{MPa}, B=709^{\circ} \mathrm{C}, C=220^{\circ} \mathrm{C}$ y $E=$ $15 \mathrm{~s}$, la resistencia máxima a la tracción alcanzó los 290,303 MPa. En las tablas I y II se muestran los resultados de los métodos Experimental (Exp.), TLBO y PSO.

Tabla I. Comparación de la máxima dureza entre los métodos Exp, TLBO y PSO 
En las tablas I y II, se observan que la máxima dureza (100,76 BHN) y resistencia máxima la tracción (278,45 MPa) obtenidas experimentalmente por Senthil y Amirthagadeswaran [34], fueron superadas por Venkata et al. [2] hasta 103,068 BHN y 290,30 MPa, respectivamente. Y con el método de optimización PSO se alcanzó una máxima dureza de 103,072 BHN y una máxima resistencia de tracción 290,303 MPa, lo que representó una mínima mejora de los resultados respecto al estudio de Venkata et al. [2].

\begin{tabular}{lccccc}
\hline \multirow{2}{*}{ Parámetros } & Exp. & TLBO & Resultados & Media & \begin{tabular}{c} 
D. \\
\cline { 4 - 6 }
\end{tabular} \\
& & & & 119 & \\
\hline Presión de compresión $(\mathrm{MPa})$ & 100 & 119 & & \\
\hline Temperatura de fusión $\left({ }^{\circ} \mathrm{C}\right)$ & 725 & 675 & 709 & \\
\hline Temperatura de compresión $\left({ }^{\circ} \mathrm{C}\right)$ & 200 & 220 & 220 & \\
\hline Tiempo de compresión $(\mathrm{s})$ & 45 & 15 & 15 & \\
\hline Máxima R. Tracción $(\mathrm{MPa})$ & 278,45 & 290,30 & $\mathbf{2 9 0 , 3 0 3}$ & 290,229 & 0,124 \\
\hline Tabla II. Comparación de la máxima resistencia a la tracción entre los métodos Exp, TLBO y \\
PSO.
\end{tabular}

Para verificar la aceptación de los resultados de la PSO en este estudio, se calculó la media y la desviación estándar para una ejecución de 50 veces el código. Las medias de la máxima dureza y de la máxima resistencia a la tracción, estuvieron muy cerca de la mejor solución, y en el caso de las desviaciones estándar, los valores indican que la dispersión entre el conjunto de la población y la media fue mínima. En la figuras III se observa cómo se alcanzaron las convergencias en la máxima dureza y resistencia a la tracción con la PSO. Las curvas indican que con el algoritmo PSO se obtuvo, a escasos números de iteraciones, la estabilidad de los resultados, y una mínima mejoría en las soluciones respecto al TLBO. El ordenador utilizado para ejecutar la PSO fue un computador con procesador Intel Core i5, y el tiempo computacional fue de 5 segundos aproximadamente, lo que es posible afirmar que el algoritmo de enjambre de partículas desarrollado es una herramienta útil para mejorar el rendimiento de plantas de fundición a compresión.

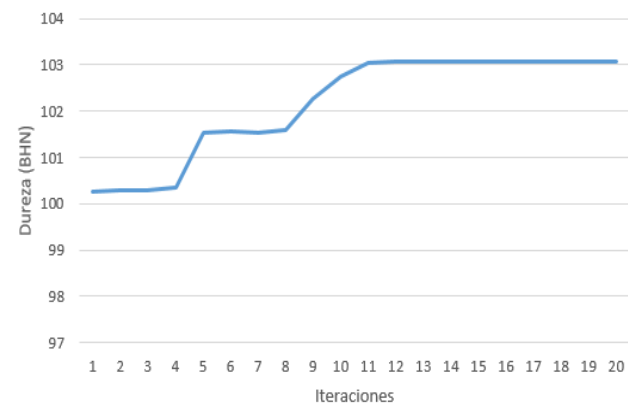

a)

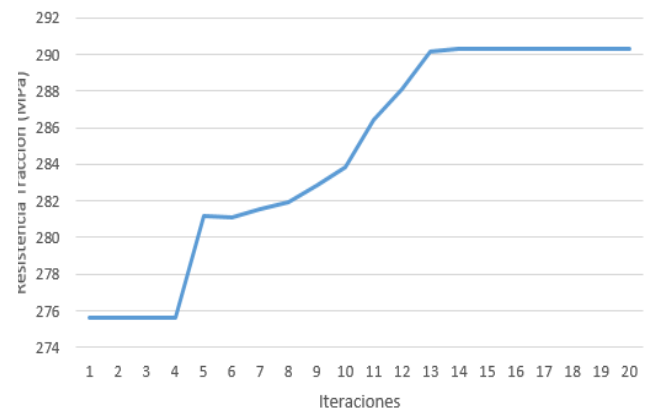

b)

Figura III. Convergencia de la PSO para a) la dureza, y b) resistencia a la tracción.

3.2 Optimización de parámetros en el proceso de fundición a presión.- Tsoukalas [35], realizó una investigación para diseñar un algoritmo de optimización de parámetros utilizando un enfoque combinado de regresión lineal múltiple y algoritmo genético (GA), para una aleación de aluminio. Las variables consideradas por Tsoukalas [35], fueron la temperatura del horno $(F)$, la temperatura del troquel $(D)$, la velocidad del émbolo en la primera etapa $(S)$, la velocidad del émbolo en la segunda etapa $(H)$ y la presión multiplicada $(M)$. En la metodología usó el método Taguchi para 
obtener el modelo matemático que se describe con la ecuación 6 , donde se relacionan los parámetros de entrada del proceso con el defecto de porosidad $(P \%)$.

$$
\begin{gathered}
P=1,623-0,766 * 10^{-3}(F)-1,301 * 10^{-3}(D)-0,136(S)+0,029(H) \\
-1,636 * 10^{-3}(M)
\end{gathered}
$$

Los rangos de operación en los parámetros del proceso fueron:

$$
\begin{gathered}
F=(610-730){ }^{\circ} \mathrm{C} \\
D=(190-270){ }^{\circ} \mathrm{C} \\
S=(0,02-0,34) \mathrm{m} / \mathrm{s} \\
H=(1,2-3,8) \mathrm{m} / \mathrm{s} \\
M=(120-280) \mathrm{bar}
\end{gathered}
$$

Utilizando un número de iteraciones de 1.000 , y sin mencionar el tamaño de la población, el algoritmo genético diseñado por Tsoukalas [35], convergió después de las 800 iteraciones. Cuando $F=729,4^{\circ} \mathrm{C}, \quad D=269,9^{\circ} \mathrm{C}, S=0,336 \mathrm{~m} / \mathrm{s}, \quad H=1,2 \mathrm{~m} / \mathrm{s}$ y $M=275,7$ Bar, la mínima porosidad alcanzada fue de $0,251 \%$.

Posteriormente, Venkata et al. [2], utilizaron los resultados obtenidos por Tsoukalas [35], y desarrollaron un algoritmo de optimización basado en enseñanza-aprendizaje (TLBO). En este algoritmo utilizó 10 iteraciones y los resultados obtenidos mostraron una mínima porosidad de $0,243 \%$ cuando $F=730{ }^{\circ} \mathrm{C}, D=270{ }^{\circ} \mathrm{C}, S=0,34 \mathrm{~m} / \mathrm{s}, H=1,20 \mathrm{~m} / \mathrm{s}$ y $M=280$ Bar.

Ahora, en el presente estudio, se utilizó el código PSO, con la finalidad de comparar los resultados obtenidos por Venkata et al. [1], y Tsoukalas [35], utilizando las restricciones y el modelo matemático descrito en la ecuación 6. Se realizaron varias ejecuciones con el algoritmo PSO, se consideró un número de 10 iteraciones y una población de $50, \mathrm{y}$ los resultados indicaron que cuando $F=730^{\circ} \mathrm{C}, D=270^{\circ} \mathrm{C}, S=0,34 \mathrm{~m} / \mathrm{s}, H=1,20 \mathrm{~m} / \mathrm{s}$ y $M=280 \mathrm{Bar}$, la mínima porosidad alcanzada por el PSO fue de $0,243 \%$. En la tabla III se muestra los detalles de los resultados.

\begin{tabular}{lccccc}
\hline \multirow{2}{*}{ Parámetros } & \multirow{2}{*}{ GA } & TLBO & \multicolumn{3}{c}{ PSO } \\
\cline { 4 - 6 } & & & Resultados & Media & D. Estándar \\
\hline Temperatura Horno $\left({ }^{\circ} \mathrm{C}\right)$ & 729,4 & 730 & 730 & & \\
\hline Temperatura Troquel $\left({ }^{\circ} \mathrm{C}\right)$ & 269,9 & 270 & 270 & & \\
\hline Veloc. Embolo 1ra. Etapa $(\mathrm{m} / \mathrm{s})$ & 0,336 & 0,34 & 0,34 & & \\
\hline Veloc. Embolo 2da. Etapa $(\mathrm{m} / \mathrm{s})$ & 1,20 & 1,20 & 1,20 & & \\
\hline Presión Múltiple (Bar) & 275,7 & 280 & 280 & & 0,021 \\
\hline Mínima Porosidad $(\%)$ & 0,251 & 0,243 & $\mathbf{0 , 2 4 3}$ & 0,248 & \\
\hline
\end{tabular}

Tabla III. Comparación de la porosidad entre los métodos GA, TLBO y PSO

En la tabla III se observa que los resultados obtenidos con el código PSO fueron exactamente igual a los reportados por Venkata et al. [2] con el algoritmo TLBO, por lo que se presume que estos resultados son la mejor solución. Para verificar la aceptación de los resultados de la PSO, se procedió a calcular la media y la desviación estándar para una ejecución de 50 veces del código. La media fue de $0,248 \%$ y la desviación estándar estuvo en 0,021 , estos valores indican que la dispersión entre el conjunto de la población y la media fue mínima. La figura IV muestra la curva de convergencia de la mínima porosidad con la PSO, en ella se observa que los resultados se estabilizan a tempranas iteraciones y el tiempo de ejecución no superó más de 4 segundos en el computador Intel Core i5. Con estos resultados, la tecnica de la PSO sin duda representa una herramienta útil para mejorar el rendimiento de plantas de fundición a presión. 


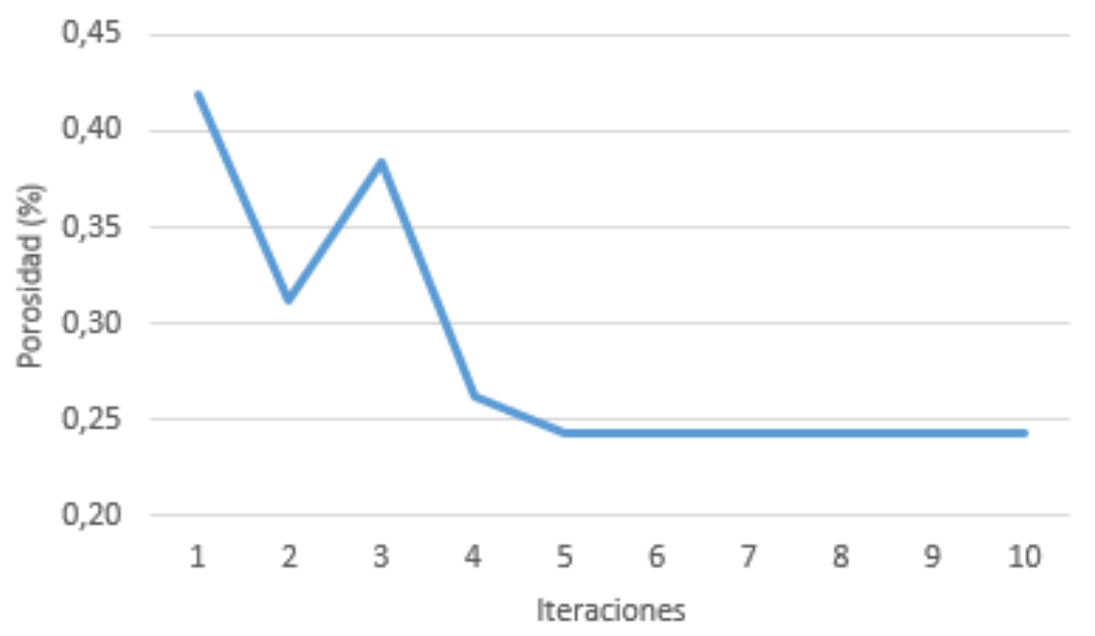

Figura IV. Comportamiento de la PSO con la porosidad.

4. Conclusiones.- En este artículo se ha desarrollo un algoritmo basado en la técnica de optimización de partículas (Particle Swarm Optimization, PSO), que simula el comportamiento social del vuelo de las bandadas de aves o el movimiento de los bancos de peces. El algoritmo PSO desarrollado ha manejado efectivamente diversos modelos matemáticos y ha demostrado buenas capacidades en el campo de la optimización de parámetros que influyen en los procesos de fundición a presión y por compresión:

- En el proceso de fundición por compresión, el algoritmo PSO consiguió en tempranas iteraciones (11 y 13) converger la máxima dureza de 103,072 BHN y la máxima resistencia a la tracción de 290,303 MPa. Estos resultados son aproximadamente igual a los obtenidos por un algoritmo de optimización de enseñanza-aprendizaje TLBO, lo que indica que el PSO es una buena alternativa para predecir las propiedades de dureza y resistencia a la tracción a un bajo costo computacional.

- En la fundición a presión, el algoritmo PSO minimizo la porosidad en la iteración 5 con un valor de $0,243 \%$, siendo el mismo resultado alcanzado mediante el algoritmo de optimización de enseñanza-aprendizaje TLBO.

- El algoritmo PSO desarrollado tiene un alto potencial para gestionar la determinación de parámetros en los procesos de fundición a presión y por compresión, sin embargo, se recomienda aplicar en otros tipos de procesos de fundición que no han sido estudiados en esta investigación. 


\section{Referencias}

[1] González-Rondón, Y. y Rengel-Hernández, J.E. (2021). Comportamiento termo fluidodinámico del acero en un molde de colada continua: una revisión, TecnoLógicas, vol. 24, nro. 51, e1856.

[2] Venkata Rao, R. Kalyankar, V.D. and Waghmare G. (2014). Parameters optimization of selected casting processes using teaching-learning-based optimization algorithm. Applied Mathematical Modelling, 38, 5592-5608.

[3] Souissi, N. Souissi, S. Le Niniven, C. Ben Amar, M. Bradai, C. and Elhalouani, F. (2014). Optimization of Squeeze Casting Parameters for 2017 A Wrought Al Alloy Using Taguchi Method. Metals, 4, 141-154; doi:10.3390/met4020141.

[4] Souissi, S. Souissi, N. Barhoumi, H. Ben Amar, M. Bradai, C. and Elhalouani, F. (2019). Characterization of the Role of Squeeze Casting on the Microstructure and Mechanical Properties of the T6 Heat Treated 2017A Aluminum Alloy. Hindawi, Advances in Materials Science and Engineering, Volume 2019, Article ID 4089537, 9 pages.

[5] Belisario, J. (2001). Evaluación de la efectividad del proceso de colada en la reducción de defectos de salpicaduras en las palanquillas producidas en la acería 150 TM de Sidor, Tesis de grado en ingeniería industrial. Universidad Nacional Experimental de Guayana

[6] Maleki, A. Shafyei, A. and Niroumand, B. (2009). Effects of squeeze casting parameters on the microstructure of LM13 alloy. Journal of Materials Processing Technology, vol. 209, no. 8, pp. 3790-3797.

[7] Dahel, A. Nogita, K. Mcdonald, S. Zindel, J. and Hogan, L. (2001). Eutectic nucleation and growth in hypoeutectic Al-Si alloys at different strontium levels. Metallurgical and Materials Transactions A, vol. 32, no. 4, pp. 949-960.

[8] Fang, Y. (2006). Mathematical modeling and experimental study of squeeze casting of magnesium alloy AM50A and aluminum alloy A356. Electronic Theses and Dissertations. 7212.

[9] Guo, H. y Yang, X. (2007). Preparation of semi-solid slurry containing fine and globular particles for wrought aluminium alloy 2024. Trans. Nonferr. Metal Soc. 2007, 17, 799-804.

[10] Han, Y.S. Kim, D.H. Lee, H.I. and Kim, Y.G. (1994). Effect of applied pressure during solidification on the microstructural refinement in an Al-Cu alloy. Scripta Metallurgica et Materialia, Vol.31, No.12, pp.1623-1628.

[11] Maeng, D.Y., Lee, J.H., Won, C.W., Cho, S.S. and Chun, B.S. (2000). The effects of processing parameters on the microstructure and mechanical properties of modified B390 alloy in direct squeeze casting. J. of Materials Processing Technology, Vol.105, pp.196-203.

[12] Hong, C.P. Shen, H.F. and Lee, S.M. (1998). Prevention of macrodefects in squeeze casting of an Al-7 wt pet Si alloy. Metallurgical and Materials Transactions B, Vol.31B, pp.297-305.

[13] Hong, C.P. Shen, H.F. and Cho, I.S. (1998). Prevention of macrosegregation in squeeze casting of an Al-4.5 wt pet Cu alloy. Metallurgical and Materials Transactions A, Vol.29A, pp.339349.

[14] Gallerneault, M. Durrant, G. and Cantor, B. (1996). The squeeze casting of hypoeutectic binary Al-Cu. Metallurgical and Materials Transactions A, Vol.27A, pp.4121-4132.

[15] Mahesh N. A. and Shrikant V. K. (2015). Optimization of die-casting process parameters using DOE. International Journal of Engineering Research and General Science Volume 3, Issue 2.

[16] Kumar, S. Kumar Gupta, A. and Chandna, P. (2012). Optimization of Process Parameters of Pressure Die Casting using Taguchi Methodology. International Journal of Mechanical, Aerospace, Industrial, Mechatronic and Manufacturing Engineering Vol:6, No:8.

[17] Ainul Haque, M. D. Babuli Kumar, J. and Dilip Kumar, M. (2017). Optimization of process parameters in cold chamber Pressure die casting using doe. International Research Journal of Engineering and Technology (IRJET). Volume: 04 Issue: 04, Apr. 
[18] Vanli, A.S. Akdogan, A.and Durakbasa, M.N. (2018). Process Optimization and Surface Modification of Die Casting AZ91 Magnesium Alloy Products. Acta physica polonica A. Vol. 134. [19] Gallardo, J. Ayala, C. y Castro, R. (2018). Control predictivo/adaptativo de sistemas complejos utilizando técnicas de ingeniería neuronal. Revista Ingenierías Universidad de Medellín, Vol. 17 Núm. 33, julio-diciembre, pp. 157-172, Medellín, Colombia.

[20] García, J. (2006). Algoritmos Basados en Cúmulos de Partículas para la Resolución de Problemas Complejos. Departamento de Lenguajes y Ciencias de la computación. Universidad de Málaga.

[21] Kennedy, J. and Eberhart, R. (1995). Particle Swarm Optimization. In Proceedings of the IEEE International Conference on Neural Networks, volume 4, pages 1942-1948, Perth, Australia, Nov. [22] Kennedy, J. and Eberhart, R. and Shi, Y. (2001). Swarm Intelligence. San Francisco: Morgan Kaufmann Publishers.

[23] Stephan, O. and Zomaya, A. (2005). Handbook Of Bioinspired Algorithms And Applications. CHAPMAN and HALL/CRC.

[24] Bäck, T. Fogel, D. and Michalewicz, Z. (1997). Handbook of Evolutionary Computation. IOP Publishing and Oxford University Press, New York and Bristol (UK), Feb.

[25] Dorigo, M. (2000). The Ant Colony Optimization Metaheuristic: Algorithms, Applications and Advances. Technical Report IRIDIA-2000-32, Universit'e Libre de Bruxelles, IRIDIA.

[26] Rao, R. Savsani, V. and Vakharia, D. (2011). Teaching-learning-based optimization: a novel method for constrained mechanical design optimization problems, Comput. Aided Des. 43, 303 315.

[27] Rao, R. Savsani, V. Vakharia, D. (2012). Teaching-learning-based optimization: an optimization method for continuous non-linear large scale problems, Inf. Sci. 183, 1-15.

[28] Rao, R. and Patel, V. (2012). An elitist teaching-learning-based optimization algorithm for solving complex constrained optimization problems, Int. J. Ind. Eng. Comput. 3 (4), 535-560.

[29] Patel, G.C.M. Krishna, P. Vundavilli, P.R. Parappagoudar, M.B. (2016). Multi-Objective Optimization of Squeeze Casting Process using Genetic Algorithm and Particle Swarm Optimization. Archives of Foundry Engineering 16(3).

[30] Shu Fu-hua. (2007). Aluminum-zinc Alloy Squeeze Casting Technological Parameters Optimization based on PSO and ANN. China Foundry, vol.4, No.3, pp.202-205.

[31] Panicker, P. and Kuriakose, S. (2020) Parameter Optimisation of Squeeze Casting Process using LM 20 Alloy: Numeral Analysis by Neural Network and Modified Coefficient-based Deer Hunting Optimization. Australian Journal of Mechanical Engineering

[32] Wu, H. Yang, X. Cao, G. Zhao, L. and Yang, L. (2021). Design and optimisation of die casting process for heavy-duty automatic transmission oil circuit board. International Journal of Cast Metals Research (IF 1.083).

[33] Patel G.C.M., Ajith B, Jonathan R, Allan D S, Aniruddh M and Ashwith M. (2018). Teaching learning based optimization of squeeze casting process for quality castings. IOP Conf. Ser.: Mater.

Sci. Eng. 376012112

[34] Senthil, P. and Amirthagadeswaran, K.S. (2012). Optimization of squeeze casting parameters for non symmetrical AC2A aluminium alloy castings through Taguchi method, J. Mech. Sci. Technol. 26 (4), 1141-1147.

[35] Tsoukalas, V. (2008). Optimization of porosity formation in AlSi9Cu3 pressure die castings using genetic algorithm analysis, Mater. Des. 29, 2027-2033.

[36] Talbi, E. G. (2009). Metaheuristics: From Design to Implementation. New Jersey, Hoboken: John Wiley \& Sons, Inc.

[37] Chun, H. L. (2012). Diseño e implementación de algoritmos aproximados de clustering balanceado en PSO. Tesis para optar al grado de Magister en ciencias mención computación. Universidad de Chile Facultad de física y matemáticas, Departamento de ciencias de la computación. 
[38] Leza, M. V. (2008). Optimización mediante Cúmulos de Partículas con Tamaño de Población Variable. Tesis de Licenciatura en Sistemas Facultad de Informática. Universidad Nacional de la Plata.

[39] Gutiérrez, D. Villa, W. y López-Lezama, J. (2017). Flujo Óptimo Reactivo mediante Optimización por Enjambre de Partículas. Información Tecnológica. Vol. 28(5), 215-224.

[40] Damiani, L. (2019). Optimización estocástica acelerada con aplicación a la ingeniería de procesos. Tesis de maestría en ingeniería de procesos petroquímicos. Universidad Nacional del Sur. 